\title{
石墨烯的功能与应用一一生长、性质与新器件
}

\author{
王斌 1 , 智林杰 1,2
}

1 国家纳米科学中心, 纳米科学卓越创新中心, 纳米系统与多级次制造重点实验室, 北京 100190

2 中国科学院大学, 北京 100049

\section{Graphene: Functions and Applications_—Growth, Properties, and New Devices}

\author{
Bin Wang ${ }^{1}$, Linjie Zhi ${ }^{1,2}$ \\ ${ }^{1}$ CAS Key Laboratory of Nanosystem and Hierarchical Fabrication, CAS Center for Excellence in Nanoscience, National Center for \\ Nanoscience and Technology, Beijing 100190, China. \\ ${ }^{2}$ University of Chinese Academy of Sciences, Beijing 100049, China.
}

Emails: wangb@nanoctr.cn (B.W.); zhilj@nanoctr.cn (L.Z.)

Published online: April 7, 2021.

石墨烯原子层厚度的二维平面结构以及其独 特的物理化学性质引起了国际学术界的广泛关 注 $^{1}$, 同时也赋予石墨烯丰富多样的光、热、电、 磁、力等功能。石墨烯的结晶度、旋转错位、物理 形变等无不引起其功能的显著变化, 而杂原子掺 杂、可设计孔洞、选择性边缘结构、特异性复合等 也往往赋予石墨烯特殊的性能与应用。同时, 在纳 米与分子尺度上对石墨烯的结构、骨架、孔道等进 行设计与构建更能衍生出许多全新的功能化石墨 烯材料, 有可能会带来科学上的重大发现。本专刊 收集了国内部分科学家在相关领域的研究成果, 通 过主题聚焦、思想碰撞来探索石墨烯独特的功能 与应用价值, 为推动石墨烯材料实际应用提供理 论基础和技术指导。本专刊分成两期在 2022 年第 1 期和第2期印刷出版。本期主要围绕高质量石墨烯 的生长、性质调控和新功能器件的开发展开讨论, 下一期则聚焦在功能化石墨烯的规模化制备和能 源相关的应用研究上。

石墨烯的功能和应用首当其冲面临的难题是 高质量石墨烯的制备。刘忠范和丁峰等 ${ }^{2}$ 首先从理 论计算的角度, 总结了各种金属祄底在石墨烯化 学气相沉积(CVD)生长过程中的各种作用与相应 的机理, 包括在催化碳裂解、修复生长过程中产生 的缺陷、以及在降温过程中石墨烯褶皱与金属表
面台阶束的形成过程等, 为实验现象提供合理的 解释, 同时为将来的实验提供可能的指导。除了金 属祄底, CVD过程中的另一个要素是气相。刘忠范 团队进一步综述了气相反应带来的影响, 包括气 相传质过程和气相反应过程, 以及在气相反应调 控下石墨烯的结晶度、洁净度、层数和生长速度等 参数的变化规律, 给 $\mathrm{CVD}$ 石墨烯的生长提供了新 的切入视角。在长期理论和实践经验积累的基础 上, 刘忠范团队在超洁净石墨烯的控制生长方面 做出了突出的贡献。他们也对此进行了总结 ${ }^{4}$, 分 析了石墨烯生长过程中表面污染物的形成机理, 综 述了超洁净石墨烯的制备方法, 列举了其优异的 性质, 并对该领域未来发展可能面临的机遇和挑 战进行了展望。

石墨烯的制备是基础, 其功能和应用则是重 要的出口。为满足不同的应用需求, 在石墨烯本征 物性的基础上, 往往还需要对其进行功能化, 但目 前对于石墨烯功能化产物缺乏系统全面的分类和 精确的定义。智林杰等 ${ }^{5}$ 在系统总结现有石墨烯功 能化研究的基础上, 给出了石墨烯功能化产物的 系统分类、各类的精确定义和相应的制备策略, 并通过典型示例进行了详细地论述。对该领域的 系统总结将有助于功能化石墨烯材料的进一步 发展。 
在功能化石墨烯的基本物性方面, 康飞宇等 6 介绍了应用于石墨烯热导率测量的微纳尺度传热 技术, 并展示了当前的理论研究成果及石墨烯在 导热材料中的应用, 包括高导热的石墨烯膜、纤维 及热界面材料等。王斌等7则从石墨烯的力学性质 出发, 总结了采用层间共价键连接的方式来克服 石墨烯层间弱作用力的策略, 以此实现石墨烯力 学性能的提升, 同时介绍了层间共价成键所采用 的方法和性质及其分析手段, 提出了下一步的发 展方向。

功能化不仅优化了石墨烯的传统物性, 也为 基于其新性质的器件开发带来了新的思路。任文 才等 8 综述了近年来石墨烯表面电荷转移掺杂的 研究现状, 着重介绍了最新发展的高效 $\mathrm{p}$ 型和 $\mathrm{n}$ 型 掺杂剂, 并探讨了基于掺杂石墨烯透明电极的高 性能光电器件的发展趋势。基于三维石墨烯组装 体材料优异的吸光和光热转化效率等性质, 曲良 体等9介绍了高效、绿色的光热水净化、收集器件, 为水资源的循环利用提供了新的途径。周建新等 ${ }^{10}$ 则围绕自供能器件的基本能量供给原理, 包括电 化学供能、光伏供能、摩擦电供能、水伏供能等, 介绍了石墨烯在自供能传感器件中的应用, 并对 基于石墨烯的自供能传感器件的未来发展、挑战 和前景进行了展望。陈成猛等 ${ }^{11}$ 将三维铜/石墨烯 气凝胶作为相变储能骨架材料, 制备了结构稳定 性高、泄漏率低的复合材料, 该类结构的构筑对未 来的相变储能、多孔微波吸收等新生研究方向有 重要的参考价值。

高质量石墨烯的制备是其高端应用的基础, 在此基础上进行功能化改性, 可以获得对其结构 和性质关系的清晰认识, 健全理论机制, 指导并创 制出新型的功能器件, 拓展石墨烯的应用前景。这 些基础研究不仅引起了学术界的广泛关注, 而且 得到了工业界的高度重视。为将功能化石墨烯材 料推向产业化, 研究人员对其规模化制备技术和 实际应用进行了长期、大量的探索, 并取得了一定 的成绩。在本专刊的下期介绍中, 我们收集了功能 化石墨烯相关的规模化制备及其在能源领域相关 应用中的一些最新进展报告。这两期的内容整体 上涵盖了石墨烯的制备理论、方法、性质、新功能 探索和规模化应用等, 全方位展现了石墨烯功能 和应用的发展现状, 希望《物理化学学报》的读者
通过阅读本专刊中的文章, 能对该领域有一些新 的认识。

\section{References}

(1) Lin, L.; Deng, B.; Sun, J.; Peng, H.; Liu, Z. Chem. Rev. 2018, 118 (18), 9281. doi: 10.1021/acs.chemrev.8b00325

(2) Cheng, T.; Sun, L. Z.; Liu, Z. R.; Ding, F.; Liu, Z. F. Acta Phys. -Chim. Sin. 2022, 38 (1), 2012006. [程婷, 孙禄钊, 刘志荣, 丁峰, 刘忠范. 物理化学学报, 2022, 38 (1), 2012006.] doi: 10.3866/PKU.WHXB202012006

(3) Chen, H.; Zhang, J. C.; Liu, X. T.; Liu, Z. F. Acta Phys. -Chim. Sin. 2022, 38 (1), 2101053. [陈恒, 张金灿, 刘晓婷, 刘忠范. 物理化学 学报, 2022, 38 (1), 2101053.] doi: 10.3866/PKU.WHXB202101053

(4) Liu, X. T.; Zhang, J. C.; Chen, H.; Liu, Z. F. Acta Phys. -Chim. Sin. 2022, 38 (1), 2012047. [刘晓婷, 张金灿, 陈恒, 刘忠范. 物理化学 学报, 2022, 38 (1), 2012047.] doi: 10.3866/PKU.WHXB202012047

(5) Ma, Y. J.; Zhi, L. J. Acta Phys. -Chim. Sin. 2022, 38 (1), 2101004. [马英杰, 智林杰. 物理化学学报, 2022, 38 (1), 2101004.] doi: 10.3866/PKU.WHXB202101004

(6) Song, H. F.; Kang, F. Y. Acta Phys. -Chim. Sin. 2022, 38 (1), 2101013. [宋厚甫, 康飞宇. 物理化学学报, 2022, 38 (1), 2101013.] doi: 10.3866/PKU.WHXB202101013

(7) Liang, T.; Wang, B. Acta Phys. -Chim. Sin. 2022, 38 (1), 2011059. [梁涛, 王斌. 物理化学学报, 2022, 38 (1), 2011059.] doi: 10.3866/PKU.WHXB202011059

(8) Ma, L. P.; Ren, W. C.; Cheng, H. M. Acta Phys. -Chim. Sin. 2022, 38 (1), 2012080. [马来鹏, 任文才, 成会明. 物理化学学报, 2022, 38 (1), 2012080.] doi: 10.3866/PKU.WHXB202012080

(9) Chen, Q.; Zhao, J.; Cheng, H. H.; Qu, L. T. Acta Phys. -Chim. Sin. 2022, 38 (1), 2101020. [陈清, 赵健, 程虎虎, 曲良体. 物理化学学 报, 2022, 38 (1), 2101020.] doi: 10.3866/PKU.WHXB202101020

(10) Hu, C.; Hu, J. B.; Liu, M. R.; Zhou, Y. C.; Rong, J. S.; Zhou, J. X. Acta Phys. -Chim. Sin. 2022, 38 (1), 2012083. [胡聪，胡俊斌, 刘梦然, 周玉成, 戎家胜, 周建新. 物理化学学报, 2022, 38 (1), 2012083.] doi: 10.3866/PKU.WHXB202012083

(11) Li, X. M.; Gao, Y. D.; Kong, Q. Q.; Xie, L. J.; liu, Z.; Guo, X. Q.; Liu, Y. Z.; Wei, X. X.; Yang, X.; Zhang, X. H.; Chen, C. M. Acta Phys. -Chim. Sin. 2022, 38 (1), 2012091. [李晓明, 高逸丹, 孔庆强, 谢莉婧, 刘卓, 郭晓倩, 刘燕珍, 卫贤贤, 杨晓, 张兴华, 陈成猛. 物理化学学报, 2022, 38 (1), 2012091.] doi: 10.3866/PKU.WHXB202012091 